\title{
Kernos
}

Revue internationale et pluridisciplinaire de religion grecque antique

8| 1995

Varia

\section{J.H. Oakley, R.H. Sinos, The Wedding in Ancient Athens}

\section{Vinciane Pirenne-Delforge}

\section{OpenEdition \\ Journals}

\section{Édition électronique}

URL : http://journals.openedition.org/kernos/624

DOI : $10.4000 /$ kernos.624

ISSN : 2034-7871

\section{Éditeur}

Centre international d'étude de la religion grecque antique

\section{Édition imprimée}

Date de publication : 1 janvier 1995

Pagination : 318-321

ISSN : 0776-3824

\section{Référence électronique}

Vinciane Pirenne-Delforge, « J.H. Oakley, R.H. Sinos, The Wedding in Ancient Athens », Kernos [En ligne], 8 | 1995, mis en ligne le 12 avril 2011, consulté le 24 septembre 2020. URL : http:// journals.openedition.org/kernos/624 ; DOI : https://doi.org/10.4000/kernos.624 
l'initiation dévoilée et parodiée). Dans les Nuées, Aristophane s'en prendrait, selon l'auteur, autant à l'orphisme de Socrate qu'à ses conceptions sophistiques. Ceci pour dire que ces cultes sont dangereux car ils remettent en cause les cultes traditionnels de la religion officielle de la fin du Ve siècle. - Gérard GoUIRAN (L'initiation dans le Roman de Jaufré) aborde la littérature initiatique du moyen âge. Roman arthurien, le roman de Jaufré parodie les thèmes chevaleresques, mais constitue néanmoins l'initiation du héros, doublé d'une réorganisation du monde.

\section{L'initiation aujourd'bui}

Le thème de l'initiation n'a pas disparu de nos sociétés modernes, elle est même encore fort prisée. Ainsi notamment dans les films westerns, comme le développe Henri AgrL (Le rite d'initiation dans le western). Le thème initiatique prépondérant dans ce cas-ci est celui du jeune garçon qui doit s'affirmer en adulte, aidé en cela par un homme d'âge mûr, à la fois gourou et protecteur, qui se retire une fois la mission accomplie. - De nos jours, le danger de la disparition de tout rituel «initiatique » entraîne de graves désordres chez les adolescents et les jeunes adultes. Marie-José BATAILle (Initiation et psychanalyse) attire notre attention sur le fait que sans point de repère, ces jeunes sont attirés par des sectes ou des reviviscences mystiques néo-nazies. Selon l'auteur, la psychanalyse peut remédier à cette absence et être assimilée à un processus initiatique. - Dans la dernière communication, Simone VIERNe (Le mythe initiatique aujourd'bui), conclut que même dans nos sociétés désacralisées le mythe initiatique est un élan créateur. Sa charge imaginaire est indispensable à l'homme pour répondre à l'angoisse de sa condition mortelle.

Ce volume d'actes est remarquable, tant au plan matériel qu'au plan scientifique. La variété des thèmes exploités (antiquité grecque, monde romain et celtique, littérature médiévale et espagnole...) et la diversité des disciplines utilisées (inscriptions, textes historiques et littéraires ...) au cours de ce colloque donnent une information complète sur les derniers développements de la recherche en matière d'initiation.

Edith GILIS

(Université de Liège)

John H. OAkLey, Rebecca H. Sinos, The Wedding in Ancient Atbens, Madison, The University of Wisconsin Press, 1993. 1 vol. 22 x $28,5 \mathrm{~cm}$, XIV+153 p., 130 fig. (Wisconsin Studies in Classics). ISBN : 0-299-13720-1.

Parmi les rituels de passage, le mariage est sans doute l'un des plus importants, pour les individus et pour la communauté. Caractérisé par des rites de séparation, de transition et d'incorporation bien connus depuis l'étude de Van Gennep sur les rites d'initiation, le temps des noces offre à l'analyse un symbolisme très riche qui permet d'appréhender à la fois le statut du couple et la nature de l'union. Les noces se situent donc au cœur de ce livre qui, pour éviter de recréer in abstracto à partir de sources très disparates une cérémonie 
qui n'a jamais existé, se concentre sur les noces athéniennes entre le VIIe et le IVe siècle avant notre ère. Pourquoi Athènes ? Parce que la documentation littéraire, épigraphique et iconographique y est abondante. Pourquoi cette période? Parce que la peinture de vases offre alors un large éventail de représentations des différentes étapes de la cérémonie.

Conjuguant ainsi toutes les sources d'information, les A. nous introduisent dans les familles athéniennes qui marient leurs enfants, depuis les «fiançailles » jusqu'à la remise de cadeaux après la nuit de noces. Textes et images se confortent ou se complètent pour offrir au lecteur un parcours clair et sensible, sous la houlette de guides bien informés des derniers développements de la recherche et qu'un réel souci pédagogique ne conduit jamais à la simplification.

Le mot gamos recouvre à la fois la noce et la relation matrimoniale qu'elle établit. Le mariage ainsi entendu est par essence la célébration d'une union sexuelle sanctionnée par la communauté qui fonde ainsi sa propre continuité. Le processus commence par le «contrat " instauré entre le père de la jeune fille et son futur beau-fils, en une poignée de mains dont un loutrophore de la fin du Ve siècle nous montre un bel exemple. Ces, « fiançailles » peuvent avoir lieu longtemps à l'avance, compte tenu de la différence d'âge entre les futurs époux. Quand vient le temps des noces, la cérémonie-type s'articule en trois journées (proaulia-gamos-épaulia), mais ce schéma peut varier.

Les préparatifs intègrent des sacrifices aux dieux et des offrandes qui marquent, pour la jeune fille, le passage à l'âge adulte. Le bain prénuptial est une opération importante qui concerne les deux fiancés et dont la description est remarquable. D'un point de vue matériel, les maisons sont décorées et le banquet préparé. La fête proprement dite comporte un repas abondant, alimenté par les sacrifices préliminaires. La célébration est familiale; les femmes et les enfants y participent, à des tables séparées. L'hôte occasionnel est souvent l'un des deux parents; un sanctuaire peut aussi bien servir de cadre à l'événement. Le menu varie selon les invités, mais des gâteaux de sésame semblent être un aliment obligé. Les danses nuptiales sont bien représentées et mettent en scène des groupes juxtaposés d'hommes et de femmes. Le dévoilement de la mariée est un moment important, qui donne lieu à un échange de cadeaux, sans que l'on sache s'il intervient pendant ou après la fête. Il est probable qu'une telle acceptation mutuelle se faisait devant témoins et avait donc lieu à l'issue du banquet, juste avant la procession menant les époux à leur nouvelle demeure. Ce transfert de la mariée de la maison de son père à celle de son mari symbolise plus particulièrement le «passage » d'un statut à l'autre, et le thème a connu une grande popularité dans la peinture de vases, surtout à figures noires. Cette imagerie mêle le plus souvent le monde divin au monde humain dans les cortèges, signifiant ainsi, tout comme les hymnes nuptiaux, que le mariage élève les nouveaux époux au-dessus de leur condition humaine. Une fois dans sa nouvelle demeure, la jeune mariée est amenée au foyer en une démarche d'incorporation affirmée en d'autres 
occasions (nouveau-nés, esclaves...). Les katachysmata, mélange de fruits secs, de dates, de figues, sont alors déversés sur le nouveau couple et certains vases font allusion au fruit que la jeune mariée doit consommer dans la maison de son époux. L'accès à la chambre nuptiale apparaît allusivement sur quelques vases, mais c'est surtout la visite matinale de la famille et des amis porteurs de cadeaux qui est illustrée.

L'analyse se termine par un bref rappel de l'apport de la peinture de vases au thème et d'un aperçu chronologique des illustrations pour évaluer les changements qui tiennent aux modes iconographiques ou à la cérémonie ellemême. Cent trente planches en noir et blanc, de très grande qualité, permettent de vérifier la pertinence de chaque description. Pour terminer, un glossaire des termes grecs utilisés, une bibliographie sélective et un index constituent autant d'instruments utiles à tous les types de lecteurs.

J.H. Oakley et R.H. Sinos offrent une synthèse bien informée, mesurée et très soignée dans la forme. On dispose aussi d'un remarquable corpus d'illustrations sur les noces, finement analysé. Et les quelques remarques et réserves qui suivent n'affectent en rien tout le bien qu'on pense de l'ouvrage : - p. 20-21 : à propos du lebes gamikos non attribué des figures 42 et 43 (Copenhagen 13113), il aurait fallu dépasser la simple référence à la décoration de la maison : cf. Fr. Lissarague, Femmes au figuré, in G. Duby, M. Perrot (éds), Histoire des femmes, 1. Antiquité, Paris, 1990, p. 174-178; - p. 31 : à propos du calyxkrater du Peintre de Pélée de la figure 74 (Ferrare, 2896), les A. adoptent l'identification Hécate pour la figure féminine portant deux flambeaux auprès du char des mariés (Thétis et Pélée); il s'agit d'un bapax iconographique et l'interprétation qu'ils donnent peut sembler trop subtile (référence à la venue d'Achille); si, comme la photographie le laisse voir, le vase ne comporte pas d'inscription, pourquoi identifier cette femme à Hécate plutôt qu'à Artémis qui porte souvent des flambeaux et constitute un des personnages divins les plus fréquemment illustrés en relation avec le mariage ? - p. 37: d'après Pollux (III, 39-40), l'apaulia serait la coutume consistant à faire dormir le marié avec une petite fille dans la maison du père de sa femme et la mariée avec un petit garçon dans la maison du père de son époux; les A. évoquent bien la présence du pais amphithales auprès des mariés, visible sur certains vases, et la couturne de la défloration prénuptiale symbolique de la fiancée; mais aucune hypothèse n'est avancée en ce qui concerne le jeune marié et la petite fille; - p. 39-40 : les A. reprennent à leur compte la plupart des hypothèse de C.M. EDwARDs (Hesperia, 53, 1984, 58-72) sur les relations entre Aphrodite Ourania et l'échelle, mais déplorent à la note 10, p. 139, que l'épithète ne soit pas attestée pour Aphrodite en relation avec les mariages : une découverte très récente est pourtant venue confirmer cette vue puisqu'on a découvert, à Athènes, un thesauros portant l'inscription stoichedon suivante (début IVe s. av. J.-C.) :

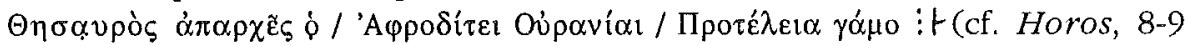
(1990-1991), p. 17-44; SEG, XLI, 182); - p. 42 : la présentation de la jeune mariée dans la phratrie de son époux comporte un sacrifice qui porte le nom 
de gamelia; il aurait fallu, fût-ce en note, évoquer l'ambiguïté des sources qui parlent aussi d'un sacrifice émanant du père pour sa fille avant le mariage (Pollux, VIII, 107); - dans la bibliographie, on peut déplorer l'absence de quelques titres utiles sur le thème: A. Avagianou, Sacred Marriage in the RItuals of Greek Religion, Bern, 1991, P. Brulé, La fille d'Athènes, Paris, 1987 et Cl. CALAME, Les chœurs de jeunes filles en Grèce archaïque, Urbino, 1977. Pour évaluer le rôle d'Aphrodite dans les mariages, qu'on me permette de renvoyer aujourd'hui à mon ouvrage L'Apbrodite grecque, Kernos Supplément 4 (1994).

Vinciane PIRENNE-DELFORGE (Université de Liège)

Ioanna Papadopoulou-Belmehd, Le chant de Pénélope. Poétique du tissage féminin dans l'Odyssée, Préface de Nicole Loraux, Paris, Belin, 1994. 1 vol. $14 \times 20,5 \mathrm{~cm}, 224$ p. (L'Antiquité au Présent). ISBN : 2-70111764-X. Prix : 125 FF.

La gloire de son mérite ne périra jamais, et les immortels composeront pour les humains un chant charmant sur la sage Pénélope. Ainsi s'exprime le poète aux vers 196-198 du chant XXIV de l'Odyssée, justifiant en quelque sorte l'intérêt que porte aujourd'hui à la femme d'Ulysse Ioanna PapadopoulouBelmehdi. Ce n'est pas la première fois que Pénélope est étudiée, mais ce livre en donne assurément l'analyse la plus soucieuse de débusquer les prétendues incohérences du personnage et de rendre un sens à l'ensemble de ses apparitions dans le poème, sans recourir à des arguments extérieurs à lui.

En 13 chapitres qui courent en continu au travers de 4 parties ( $L$ 'Ithaque de Pénélope; Tisserandes de l'utopie; le rituel est poétique; Le tissage de la matière épique), l'A. nous présente l'équivalent féminin d'Ulysse le héros: Pénélope, la reine rusée qui, par les mouvements alternatifs du tissage et du détissage, va permettre à la mémoire du roi de survivre dans l'île d'Ithaque qui croit à sa mort obscure. Volontairement immobilisée dans le statut ambigu de la jeune fille adonnée aux travaux d'Athéna, la reine est un faux-semblant de vierge, elle qui tente de protéger son fils et attend le retour de son époux. Le refus du mariage avec l'un des prétendants n'est pas le signe de sa velléité mais celui de sa détermination. C'est grâce à elle et à son combat contre la perte de la mémoire qu'Ulysse pourra retrouver son identité et sa royauté, tout en fondant son kleos, sa gloire.

Le thème du tissage, très finement analysé par l'auteur, est ambivalent : d'un côté, il peut être utilisé comme métaphore de la fonction royale, de l'autre il est omniprésent dans ce monde irréel de personnages féminins que doit traverser Ulysse pour rentrer au foyer. Le tissage, associé à Pénélope la vú $\mu \varphi \eta$ tout autant qu'aux nymphes éternelles que sont Calypso, Circé, Nausicaa ou encore les déesses de la grotte en Ithaque, est le noud du passage d'un monde à l'autre, dans le dédale du parcours d'Ulysse autant que dans le palais intemporel parce que figé sur un problème matrimonial. 